\title{
Iterative estimation of undesired signal power for superposed multicarrier transmission with channel estimation error
}

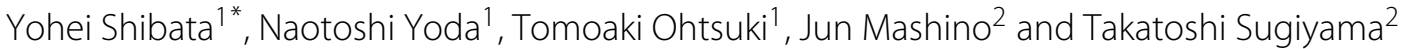

\begin{abstract}
Superposed multicarrier transmission scheme is known to improve frequency utilization efficiency when several wireless systems share the same spectrum. To suppress the effect of interference, forward error correction (FEC) metric masking is proposed. In this technique, the log-likelihood ratio (LLR) that corresponds to the superposed band is set to zero, because received bits that correspond to the superposed band are unreliable. However, to apply FEC metric masking, the information about superposed band must be known at the receiver beforehand. Furthermore, the received bits contain channel estimation errors, which are the cause of performance degradation. In this paper, we propose an iterative estimation technique for undesired signal power (noise, interference, and channel estimation error) for superposed multicarrier transmission. We use the estimated power of the undesired signal to calculate the LLR that takes the channel estimation error into account, since including this extra information about the channel improves the bit error rate (BER). The proposed scheme estimates the power of undesired signal on each subcarrier, and thus, the information about superposed band is not required. Simulation results show that the accuracy of estimating undesired signal becomes more reliable as the number of estimations increases, so that BER becomes better as a result of iterative estimation.
\end{abstract}

Keywords: Superposed multicarrier transmission; Channel estimation error; LLR

\section{Introduction}

Because of the diversification of wireless systems and the growing demand for the spectrum, deficiency in spectrum resources is a problem. To improve frequency utilization efficiency, superposed multicarrier transmission, where several independent wireless systems share the same frequency band, is proposed [1]. The technique requires a narrower frequency band than traditional spectrum allocation, because each spectrum is overlapped without guard bands as shown in Figure 1. However, desired signal suffers from interference from other systems.

In 5th generation (5G) wireless system, multi-RAT (radio access technology) where several systems coexist is assumed. 5G wireless systems are not necessarily operated in a dedicated band, such as long-term evolution

\footnotetext{
*Correspondence: shibata@ohtsuki.ics.keio.ac.jp

1 Department of Information and Computer Science, Keio University, 3-14-1, Hiyoshi, Kohoku-ku, Yokohama 223-8522, Japan

Full list of author information is available at the end of the article
}

(LTE) advanced in unlicensed band, so frequency allocation where each system is overlapped in frequency domain is conceivable. Thus, we need to suppress the effect of interference without coordination between multi-RATs.

To mitigate the effects of interference, forward error correction (FEC) metric masking is proposed [1]. FEC metric masking replaces log-likelihood ratios (LLRs) of the bits that suffer from interference with zero in order not to trust the received bits, and zero is the most ambiguous value. In other words, the probabilities when a received bit is zero and one are the same. The LLRs under interference can be wrong; therefore, using wrong LLR results in degradation of bit error rate (BER).

However, applying FEC metric masking requires the information of superposed band in advance. Therefore, the superposed band detection techniques are proposed under superposed multicarrier transmission in [2-4]. A superposed band detection technique is proposed in [2] where the superposed band is detected by searching the FEC metric masking position that minimizes the packet

\section{Springer}

(c) 2015 Shibata et al: licensee Springer. This is an Open Access article distributed under the terms of the Creative Commons Attribution License (http://creativecommons.org/licenses/by/4.0), which permits unrestricted use, distribution, and reproduction in any medium, provided the original work is properly credited. 

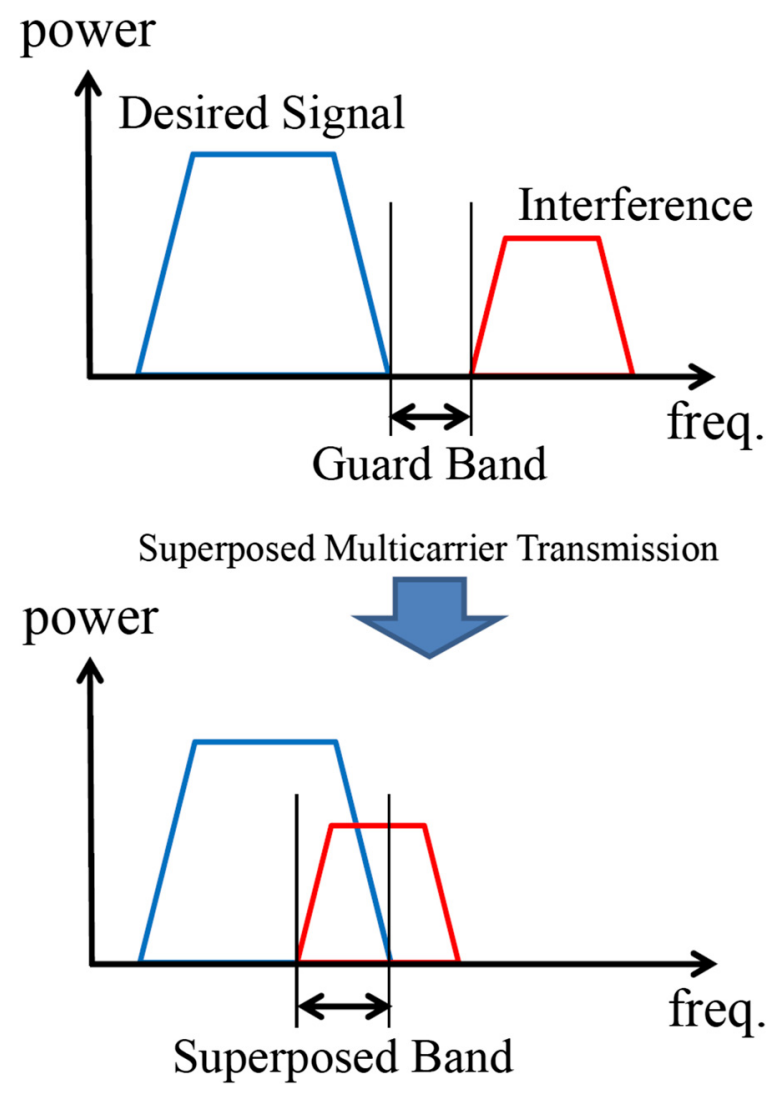

Figure 1 Spectrum allocation in superposed multicarrier transmission.

error rate (PER). This scheme can detect the superposed band but requires a large computational complexity, because the receiver needs to perform turbo decoding several times. In [3], superposed band is detected based on residual power calculated by subtracting replica signals from received ones. Since the received signals on superposed band are affected by interference, the residual power on the superposed band is larger than that of on non-superposed band. By exploiting the difference of residual power between superposed and nonsuperposed bands, superposed bands are detected. This scheme can reduce computational complexity compared to [2], because it requires decoding only once.

In addition, a conventional scheme, such as FEC metric masking, does not take into account the channel estimation error for calculating LLRs. Channel estimation error is known to cause intersymbol interference (ISI) and degradation of BER performance [5,6]. Therefore, it is required to compensate the effect of channel estimation error. In $[7,8]$, LLR that takes into account the channel estimation error is proposed under single carrier transmission. It is shown that considering channel estimation error for LLR results in better BER performance.
In this paper, we propose an iterative estimation technique for undesired signal power (noise, interference, and channel estimation error) and used it for LLR setting considering channel estimation error. In addition, we clarify the effects of the LLR setting on BER on superposed multicarrier transmission. We use the estimated power of the undesired signal for calculating LLR that takes into account the channel estimation error. Since the related works did not consider channel estimation error, our scheme is different in that we consider channel estimation error for superposed multicarrier transmission. The proposed scheme estimates the power of the undesired signal on each subcarrier, and thus, the information about superposed band is not required. Our proposed scheme does not require coordination from other systems, so it can be applied to a multi-RAT situation. Simulation results show that the BER of the proposed scheme when the iteration number of estimation is five becomes better than that when the iteration number is one.

\section{System model}

We assume an orthogonal frequency division multiplexing (OFDM) system with single transmit and single receive antennas. Each OFDM symbol, which has $L$ subcarriers, contains FEC blocks generated by a turbo encoder. At the receiver, after the removal of the guard interval of the OFDM symbol at time $t$, the $L^{\prime}$ point fast Fourier transform (FFT) is applied to it to obtain the $L^{\prime} \times 1$ frequency domain OFDM symbol. $L$ entries are extracted from the $L^{\prime} \times 1$ vector. The received signal at subcarrier index $l$ $(l=0,1, \cdots, L-1)$ at time $t$ is given by:

$y(t, l)= \begin{cases}h(t, l) x(t, l)+i(t, l)+n(t, l), & \text { for superposed band } \\ h(t, l) x(t, l)+n(t, l), & \text { for non-superposed band }\end{cases}$

where $h(t, l), x(t, l), i(t, l)$, and $n(t, l)$ are channel coefficient, transmit signal, interference, and noise components, respectively. Interference and noise components are white circular Gaussian random variables with probability distributions $\mathcal{C N}\left(0, \sigma_{\text {if }}^{2}\right), \mathcal{C N}\left(0, \sigma_{n}^{2}\right)$, respectively.

Figure 2 shows an example of the packet structure. Each packet has two pilot symbols followed by several data symbols. In our simulation, we set the number of data symbols 5 . Since $x=1$ is assumed to be sent as a pilot symbol, the received signals corresponding to two pilot signals are expressed as:

$$
y_{i}(t, l)=h_{i}(t, l)+n_{i}(t, l)+i_{i}(t, l) . i=1,2 .
$$

Furthermore, the power of the received signal is normalized to 1 , and SNR is defined as follows:

$$
\mathrm{SNR}=\frac{1}{\sigma_{n}^{2}}
$$




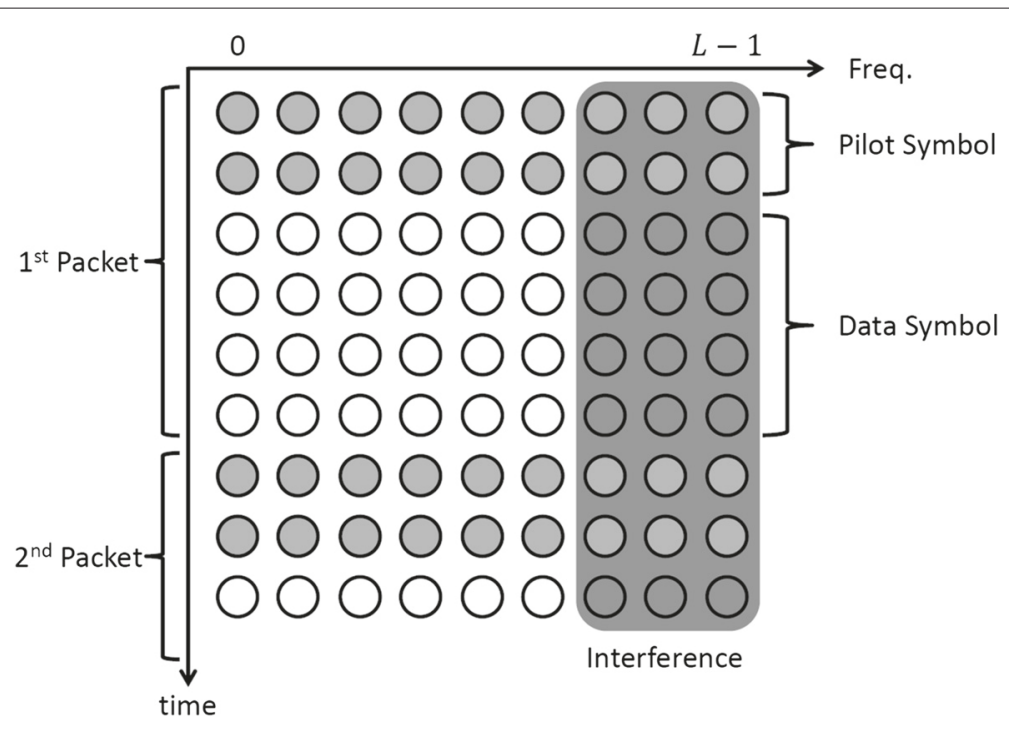

Figure 2 Packet structure.

In this paper, we assume a block-fading channel, and we disregard channel fluctuations within a packet. Thus, we approximate $h_{1} \approx h_{2}$, and the channel is estimated by taking an average of two pilot symbols on each subcarrier.

$$
\hat{h}(l)=\frac{1}{2} \sum_{i=1}^{2} y_{i}(t, l) .
$$

However, channel estimation $\hat{h}$ is not perfect due to noise and interference. Thus, $\hat{h}$ is written using channel estimation error $e$ [7].

$$
\hat{h}=h+e .
$$

$e$ is an independent, zero mean, complex Gaussian random variable, with variance of $\sigma_{e}^{2}=\mathbf{E}\left[e e^{*}\right]$. Both $y(t, l)$ and $\hat{h}(t, l)$ are given as inputs to the turbo decoder to compute LLRs. The LLR of the $m$-th bit $c(t, l, m)$ of the data symbol at time $t$, at the $l$-th subcarrier is given by:

$$
\operatorname{LLR}[c(t, l, m)]=\ln \left[\frac{p[c(t, l, m)]=1}{p[c(t, l, m)]=0}\right] .
$$

Under Gaussian noise without any interference signals, Equation 6 is rewritten as follows.

$\operatorname{LLR}^{\mathrm{Conv}}[c(t, l, m)]=\ln \left[\frac{\sum_{x \in X_{1}(m)} \frac{1}{\pi \sigma_{n}^{2}} \exp \left(-\frac{|y(t, l)-x(t, l) \hat{h}(t, l)|^{2}}{\sigma_{n}^{2}}\right)}{\sum_{x \in X_{0}(m)} \frac{1}{\pi \sigma_{n}^{2}} \exp \left(-\frac{|y(t, l)-x(t, l) \hat{h}(t, l)|^{2}}{\sigma_{n}^{2}}\right)}\right]$, where $X_{1}(m)$ and $X_{0}(m)$ are element sets of phase-shift keying (PSK) or quadrature amplitude modulation (QAM) with the $m$-th bit equals one and zero, respectively. This LLR assumes perfect channel estimation. However, BER performance degrades because channel estimation $h$ contains channel estimation error as shown in Equation 5.

The conventional scheme such as FEC metric masking does not consider channel estimation error and uses Equation 7 as LLR. Therefore, under circumstances with interference, LLR is not set correctly due to interference and channel estimation error. Conventional schemes [2-4] detect superposed band and estimate desired to undesired signal power ratio (DUR), where they do not estimate channel estimation error so that the LLR is set without taking channel estimation error into account. In this paper, we propose an iterative estimation technique for undesired signal power (noise, interference, and channel estimation error) for the purpose of calculating LLR in [7] given by Equation 8 .

$\operatorname{LLR}[c(t, l, m)]=\left\{\begin{array}{l}\ln \left[\frac{\sum_{x \in X_{1}(m)} \exp \left(-\frac{|y(t, l)-x(t, l) \hat{h}(t, l)|^{2}}{|x(t, l)|^{2} \sigma_{e}^{2}+\sigma_{n}^{2}+\sigma_{i f}^{2}}\right)}{\sum_{x \in X_{0}(m)} \exp \left(-\frac{|y(t, l)-x(t, l) \hat{h}(t, l)|^{2}}{|x(t, l)|^{2} \sigma_{e}^{2}+\sigma_{n}^{2}+\sigma_{i f}^{2}}\right)}\right], \\ \text { for superposed band } \\ \ln \left[\frac{\sum_{x \in X_{1}(m)} \exp \left(-\frac{|y(t, l)-x(t, l) \hat{h}(t, l)|^{2}}{|x(t, l)|^{2} \sigma_{e}^{2}+\sigma_{n}^{2}}\right)}{\sum_{\substack{x \in X_{0}(m) \\ \text { for non-superposed band }}} \exp \left(-\frac{|y(t, l)-x(t, l) \hat{h}(t, l)|^{2}}{|x(t, l)|^{2} \sigma_{e}^{2}+\sigma_{n}^{2}}\right)}\right] .\end{array}\right.$ 
With this scheme, the power of the undesired signal is estimated on each subcarrier, and the LLR is calculated using the estimated power of undesired signal $\hat{\sigma}^{2}(l)$.

In addition, we define a superposed rate $\alpha$ in this paper. This is defined as follows.

$$
\alpha=\frac{\text { Number of affected subcarriers }}{\text { Number of all subcarriers }} \text {. }
$$

\section{Proposed scheme}

We propose an iterative estimation technique for undesired signal power for superposed multicarrier transmission. The power of the undesired signal is estimated based on residual power, which is derived by subtracting a replica signal from the received one. In this section, we describe how to estimate the power of undesired signal in detail.

Figure 3 shows the proposed receiver structure. When a packet is received at the receiver, we have no interference information at first. Thus, the initial LLR is calculated with Equation 7 that does not consider interference, and we have to estimate noise variance. As mentioned in Equation 2, a packet has two pilot symbols on each subcarrier. Since two pilot symbols are adjacent in time domain and we assume low-speed fading, we approximate $h_{1} \approx h_{2}$. Thus, on the non-superposed subcarriers, we have:

$$
\begin{aligned}
e(l) & =y_{1}(t, l)-y_{2}(t, l) \\
& =h_{1}(t, l)-h_{2}(t, l)+n_{1}(t, l)-n_{2}(t, l) \\
& \simeq n_{1}(t, l)-n_{2}(t, l) .
\end{aligned}
$$

Noise is estimated by taking the variance of $\mathbf{e}$, where $\mathbf{e}=[e(0) e(1) \cdots e(L-1)]$, and the size of $\mathbf{e}$ is equal to the number of subcarriers, and it is written as follows.

$$
\hat{\sigma}_{n}^{2}=\operatorname{var}[\mathbf{e}]
$$

This might be affected by interference on the superposed subcarriers and $e(l) \simeq n_{1}(t, l)-n_{2}(t, l)+i_{1}(t, l)-$ $i_{2}(t, l)$ since e contains elements that suffer from interference. However, the effect of interference is averaged over all subcarriers and becomes small when the superposed rate is not so high. Using this estimated noise and channel derived in Equation 4, the received bits are decoded.

Secondly, the replica signal is made using the output bit sequence $\hat{x}$ and the channel estimation $\hat{h}$. Then, the replica signal is subtracted from the received one. If the received signal is decoded correctly, $\hat{x}=x$, we have:

$$
\begin{aligned}
e^{\prime}(t, l)=y(t, l)-\hat{h} \hat{x} & =h x+n-(h+e) x \\
& =h x+n-h \hat{x}-e x \\
& =n-e x .
\end{aligned}
$$

In Equation 12, $e^{\prime}(t, l)$ stands for the undesired signal component at time $t$ and on subcarrier index $l$. Thus, the power of undesired signal $\hat{\sigma}^{2}(l)$ is derived by taking the variance of $\mathbf{e}^{\prime}(l)$ on each subcarrier, where $\mathbf{e}^{\prime}(l)=$ $\left[e^{\prime}\left(t^{\prime}, l\right), e^{\prime}\left(t^{\prime}+1, l\right), \cdots, e^{\prime}\left(t^{\prime}+m-1, l\right)\right]$. Here, $\mathbf{e}^{\prime}(l)$ is the vector of residual signal on subcarrier index of $l . m$ is the number of data symbol on each subcarrier, and the size of $\mathbf{e}^{\prime}(l)$ is $m$. Since the power of the undesired signal is different between the superposed bands and non-superposed bands, the estimated power of undesired signal is given by Equation 13. Furthermore, since $\hat{\sigma}^{2}(l)$ is estimated on each subcarrier, it can take narrow band interference into consideration.

$$
\hat{\sigma}^{2}(l)= \begin{cases}\hat{\sigma}_{n}^{2}+\hat{\sigma}_{e}^{2}, & \text { for non-superposed band } \\ \hat{\sigma}_{n}^{2}+\hat{\sigma}_{i f}^{2}+\hat{\sigma}_{e}^{2} . & \text { for superposed band }\end{cases}
$$

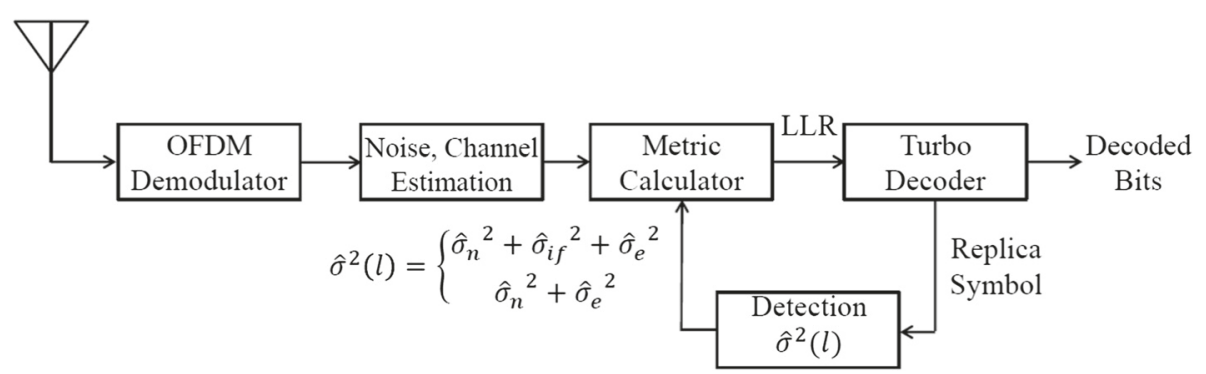

Figure 3 Proposed receiver structure. 
With the power of undesired signal $\hat{\sigma}^{2}(l)$, the LLR is recalculated by Equation 14.

$\operatorname{LLR}^{\operatorname{Prop}}[c(t, l, m)]=\ln \left[\frac{\sum_{x \in X_{1}(m)} \exp \left(-\frac{|y(t, l)-\hat{h}(t, l) x(t, l)|^{2}}{\hat{\sigma}^{2}(l)}\right)}{\sum_{x \in X_{0}(m)} \exp \left(-\frac{|y(t, l)-\hat{h}(t, l) x(t, l)|^{2}}{\hat{\sigma}^{2}(l)}\right)}\right]$.

Furthermore, the decoded bits are fed into "detection $\hat{\sigma}^{2}(l)$ " in Figure 3 again. By repeating this estimation process, the estimation accuracy of $\hat{\sigma}^{2}(l)$ could be better.

\section{Performance evaluation}

Table 1 lists major parameters used for the computer simulation. The parameters are chosen in accordance with [2]. The proposed scheme with the LLR that takes into account the channel estimation error is compared to the scheme with the LLR given by Equation 7 and FEC metric masking. The LLR of FEC metric masking is written as follows.

$\operatorname{LLR}^{Z e r o}[c(t, l, m)]=\left\{\begin{array}{l}0, \\ \text { for superposed band } \\ \ln \left[\frac{\sum_{x \in X_{1}(m)} \frac{1}{\pi \sigma_{n}^{2}} \exp \left(-\frac{|y(t, l)-x(t, l) h(t, l)|^{2}}{\sigma_{n}^{2}}\right)}{\sum_{x \in X_{0}(m)} \frac{1}{\pi \sigma_{n}^{2}} \exp \left(-\frac{|y(t, l)-x(t, l) h(t, l)|^{2}}{\sigma_{n}^{2}}\right)}\right] . \\ \text { for non-superposed band }\end{array}\right]$.

Table 1 Simulation parameters

\begin{tabular}{ll}
\hline Parameters & \\
\hline Modulation & QPSK/OFDM \\
Number of data subcarriers $L$ & 62 \\
Symbol duration & $4 \mu \mathrm{s}$ \\
FEC & Turbo code, code rate: 1/2 \\
Decoding algorithm & Linear-log-MAP \\
Channel model & Multipath Rayleigh fading \\
Doppler frequency & $30 \mathrm{~Hz}$ \\
Power delay profile & $1 \mathrm{~dB}$ exponential decaying model \\
Packet & Two pilot symbols + five data symbols \\
DUR & $6,3,0 \mathrm{~dB}$ \\
Superposed rate $\alpha$ & $10 / 62,16 / 62$ \\
\hline
\end{tabular}

The power of the undesired signal and the information about the superposed band are given for Equations 7 and 15 , and interference and channel estimation errors are not considered in both cases. Channel estimation is assumed to be perfect for Equations 7 and 15. On the other hand, the proposed scheme uses the estimated power of undesired signal which takes the channel estimation error into account. In [1], channel estimation is assumed to be perfect. In this paper, we consider channel estimation error as mentioned in Equation 5.

\section{Effects of channel estimation errors}

First, we examine the effects of channel estimation errors when the superposed rate is $\alpha=0 / 62,10 / 62$. Figure 4 shows a BER comparison between the scheme with LLR given by Equation 14 and LLR given by Equation 16 . Although Equation 16 is set in accordance with the power of noise and interference, it does not take into account the channel estimation errors. However, Equation 14 considers channel estimation errors. In both cases, the power of the undesired signal and information about the superposed band are known (estimation is perfect), and LLR is set properly according to Equations 14 and 16.

From the figure, we can see that the scheme with the LLR that takes into account the channel estimation errors performs better than the scheme that does not. In our scheme, the $E_{b} / N_{0}$ improvement is about $0.5 \mathrm{~dB}$ at $10^{-4} \mathrm{BER}$ in the figure. In [7], the $E_{b} / N_{0}$ improvement is smaller than $1 \mathrm{~dB}$ at $10^{-4} \mathrm{BER}$, which is comparable to the improvement in our scheme. Therefore, we conclude that considering channel estimation for LLR is necessary.

$\operatorname{LLR}[c(t, l, m)]=\left\{\begin{array}{l}\ln \left[\frac{\sum_{x \in X_{1}(m)} \frac{1}{\pi\left(\sigma_{n}^{2}+\sigma_{i f}^{2}\right)} \exp \left(-\frac{|y(t, l)-x(t, l) \hat{h}(t, l)|^{2}}{\sigma_{n}^{2}+\sigma_{i f}^{2}}\right)}{\sum_{x \in X_{0}(m)} \frac{1}{\pi\left(\sigma_{n}^{2}+\sigma_{i f}^{2}\right)} \exp \left(-\frac{|y(t, l)-x(t, l) \hat{h}(t, l)|^{2}}{\sigma_{n}^{2}+\sigma_{i f}^{2}}\right)}\right], \\ \text { for superposed band } \\ \ln \left[\frac{\sum_{x \in X_{1}(m)} \frac{1}{\pi \sigma_{n}^{2}} \exp \left(-\frac{|y(t, l)-x(t, l) \hat{h}(t, l)|^{2}}{\sigma_{n}^{2}}\right)}{\sum_{x \in X_{0}(m)} \frac{1}{\pi \sigma_{n}^{2}} \exp \left(-\frac{|y(t, l)-x(t, l) \hat{h}(t, l)|^{2}}{\sigma_{n}^{2}}\right)}\right] . \\ \text { for non-superposed band }\end{array}\right]$

\section{Estimated power of undesired signal versus the number of iterations}

Figures 5 and 6 show root mean square error (RMSE) of the estimated power of undesired signal on nonsuperposed bands and superposed bands, respectively. 


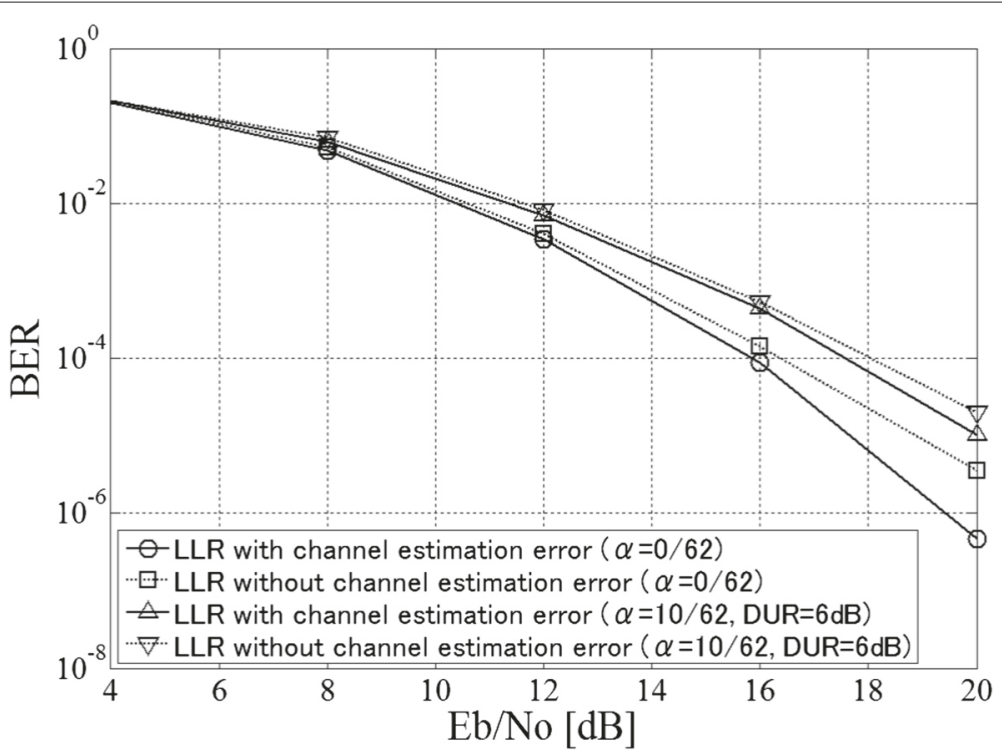

Figure 4 BER comparison with or without channel estimation error where superposed bands, $\sigma_{n}^{2}, \sigma_{i f}^{2}$, and $\sigma^{2}(I)$, are known.

RMSE is calculated using the averaged value on superposed band and non-superposed band. In those figures, the horizontal axis stands for how many times the estimation process of $\hat{\sigma}^{2}(l)$ is executed.

Figure 5 shows the RMSE of the estimated power of undesired signal on non-superposed band. We can see that the estimated value gets closer to 0 as the number of estimation increases. When DUR $=6 \mathrm{~dB}$, the RMSE value is smaller than that when DUR $=0,3 \mathrm{~dB}$. This is because the initial decoded bits when DUR $=6 \mathrm{~dB}$ are more reliable than those when DUR $=0,3 \mathrm{~dB}$. Even though DUR is low, the RMSE becomes close to zero within five iterations.
Figure 6 shows the RMSE of the estimated power of undesired signal on superposed band for each DUR. In the figure, we can see that the RMSE value when DUR is high is smaller than that when DUR is low. This is because, when DUR $=6 \mathrm{~dB}$, since the power of interference is not so large, the estimated power is calculated reliably from the beginning. We can also see that the improvement of RMSE on superposed band is limited since RMSE converges within iteration number of two.

In short, the accuracy of estimated power of undesired signal becomes comparatively reliable within iteration number of five.

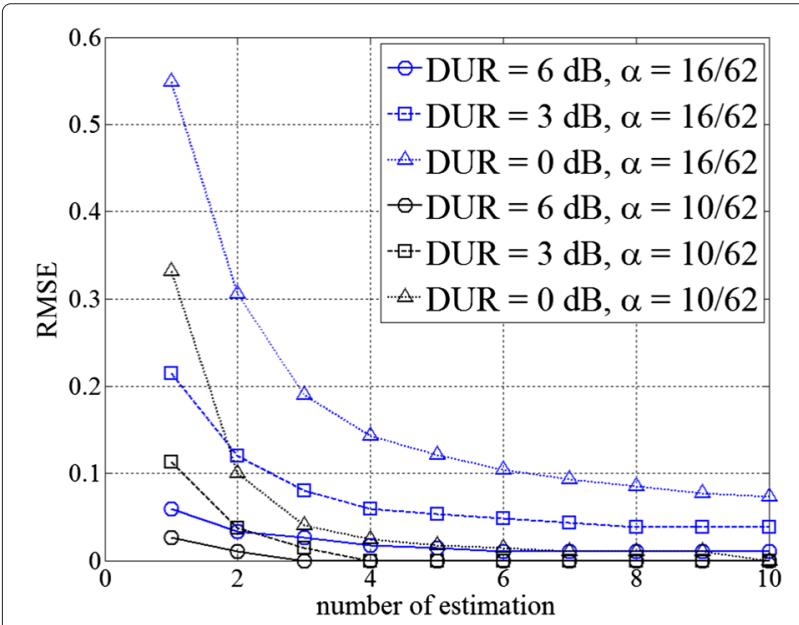

Figure 5 RMSE of the estimated power of undesired signal on non-superposed band, $\alpha=16 / 62, E_{b} / N_{0}=24 \mathrm{~dB}$.

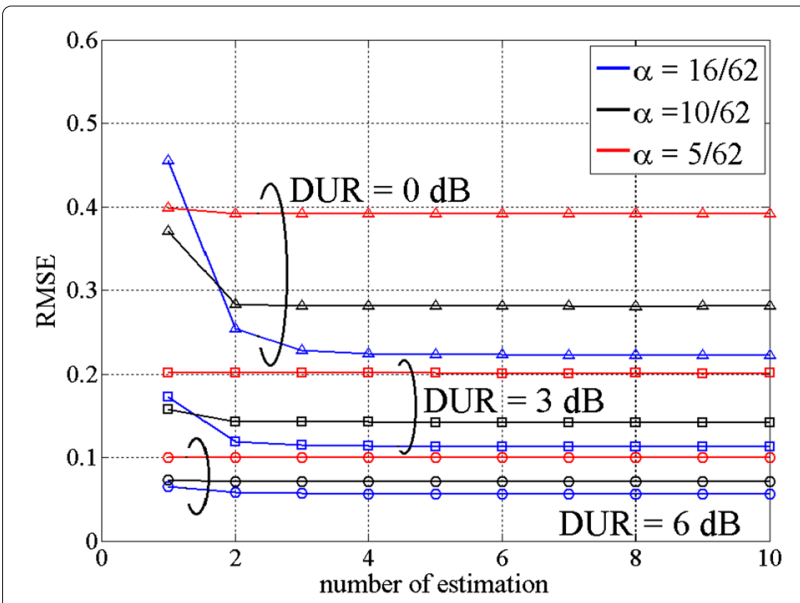

Figure 6 RMSE of the estimated power of undesired signal on superposed band, $E_{b} / N_{0}=24 \mathrm{~dB}$. 


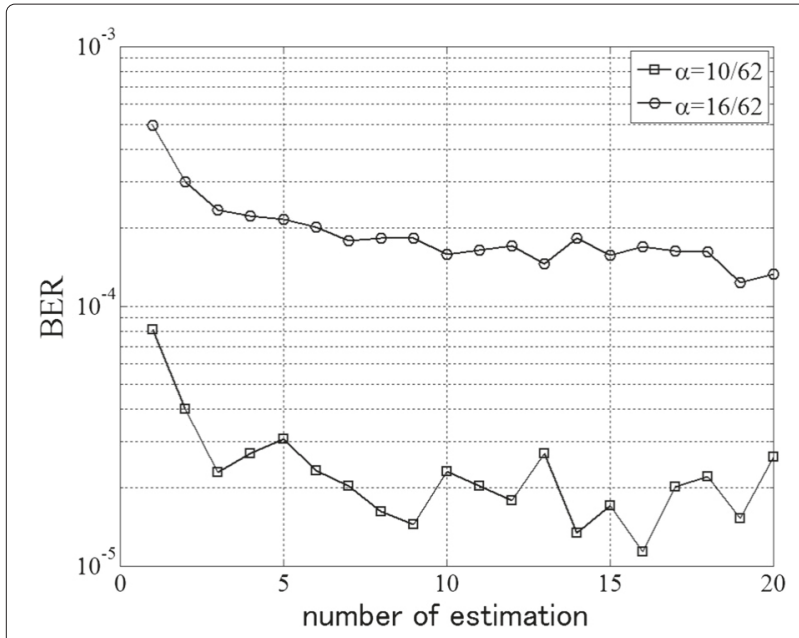

Figure $7 \mathrm{BER}$ versus number of estimation $E_{b} / N_{0}=24 \mathrm{~dB}, \mathrm{DUR}=$ $6 \mathrm{~dB}$.

\section{BER versus the number of estimations of the power of undesired signal}

Figures 7 shows the relationship between BER and the iteration number of estimation of the power of undesired signal at $E_{b} / N_{0}=24 \mathrm{~dB}$ for $\alpha=16 / 62,10 / 62$, respectively. From the figure, we can see that the BER becomes better as the number of estimations increases. This tendency is the same for both $\alpha=10 / 62$ and $\alpha=16 / 62$. We can also see that the BER hits the bottom at a certain number of estimations. In other words, a large number of estimations of the undesired signal power do not necessarily result in better BER performance. Similar to the accuracy of the estimated power of the undesired signal, the BER also almost converges at about five iterations. Thus, we set the iteration number to five as the required number of estimations of the power in the following evaluation.

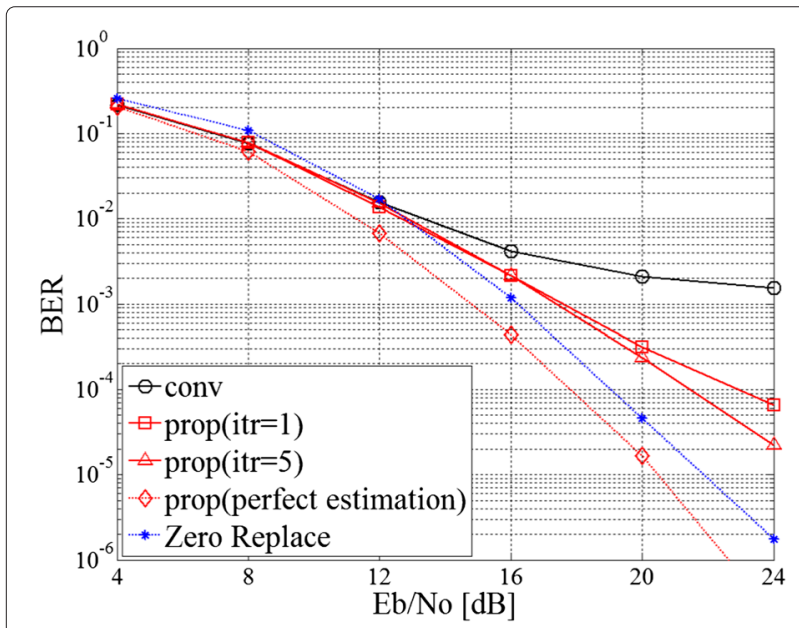

Figure $8 \mathrm{BER}, \alpha=10 / 62$, DUR $=6 \mathrm{~dB}$.

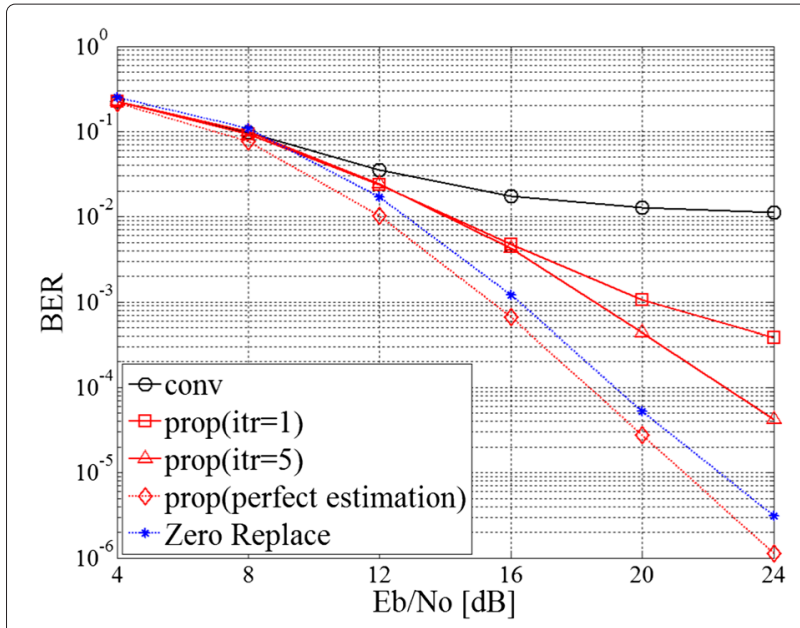

Figure $9 \mathrm{BER}, \alpha=10 / 62$, DUR $=3 \mathrm{~dB}$.

\section{LLR scheme comparison by BER performance}

Figures 8, 9, 10, 11, 12, and 13 show the BER performances of the conventional scheme with the LLR given by Equation 7, FEC metric masking, and the proposed scheme when $\alpha=10 / 62,16 / 62$ and DUR $=0,3,6$ $\mathrm{dB}$, respectively. The power of noise is known for "conv" and "Zero Replace", and the superposed band is also known for "Zero Replace", while the proposed scheme is given no information in advance. In the figures, "conv" stands for the scheme with the LLR that only considers noise and does not consider interference and channel estimation, which is given by Equation 7. "Zero Replace" stands for FEC metric masking [1] that uses the LLR given by Equation 15. "perfect estimation" is when estimation of the power of undesired signal is perfect. "itr" stands for the iteration number of estimation of the power.

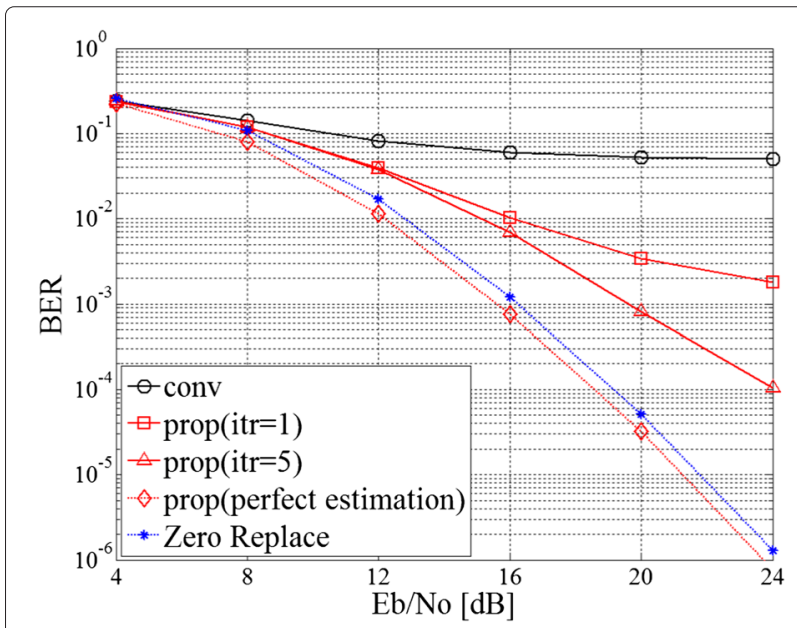

Figure $10 \mathrm{BER}, \alpha=10 / 62$, DUR $=0 \mathrm{~dB}$. 


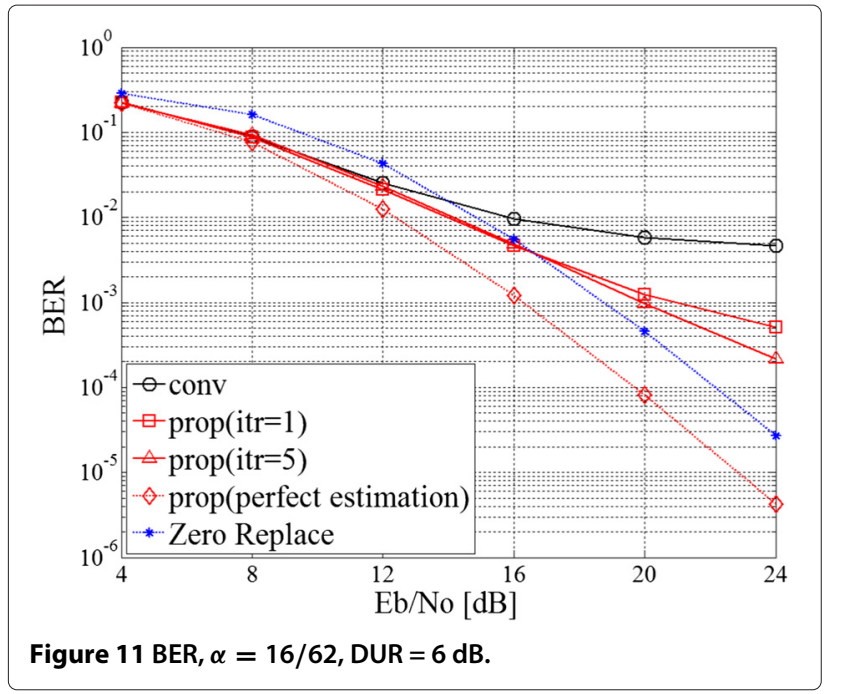

The BER of the proposed scheme is better than that of "conv", because the LLR of the proposed scheme is calculated with the estimated power of noise, interference, and channel estimation error. Furthermore, the BER of the proposed scheme when the estimation is iterated five times is better than that of once.

In terms of DUR, when the DUR is high, the BER of the proposed scheme is better than that when DUR is low. This is because initial decoded bits have less error bits when the DUR is high than when DUR is low.

When the superposed rate $\alpha$ is low, the BER becomes better than that when $\alpha$ is high. This is because the number of reliable LLRs is larger when $\alpha$ is low since the accuracy of estimation for the power of undesired signal is good on non-superposed subcarriers.

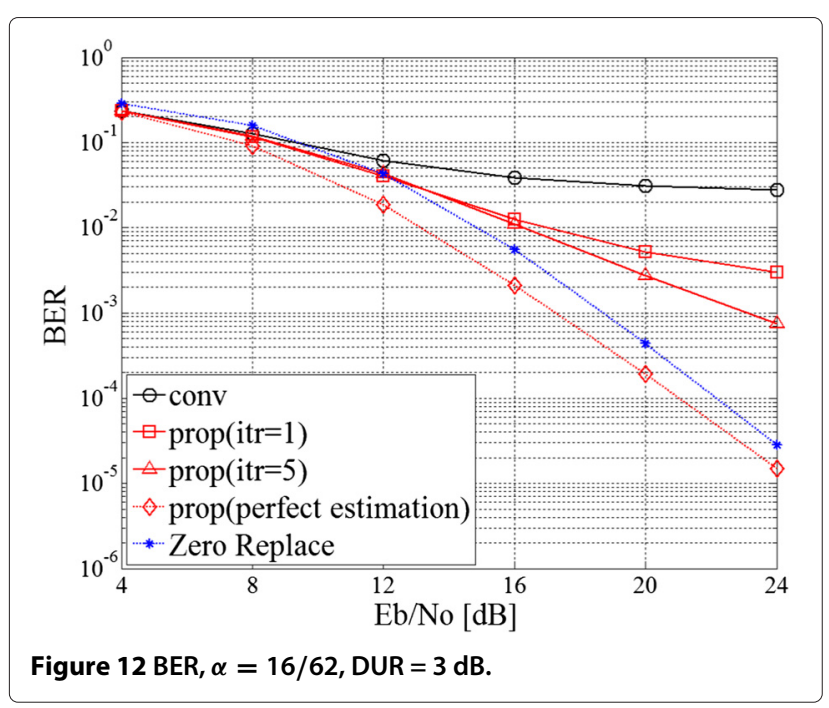

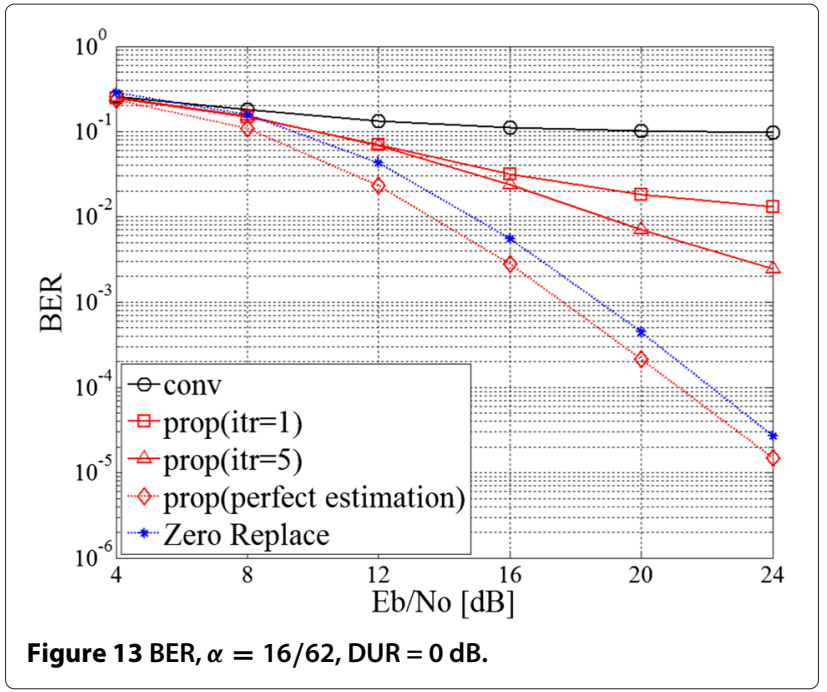

In conclusion, the BER of the proposed scheme becomes better as a result of iterative estimation of the power of undesired signal.

\section{Conclusions}

In this paper, we propose an iterative estimation technique for undesired signal power for superposed multicarrier transmission. We use a LLR that takes into account the channel estimation errors to mitigate their effects. The proposed technique estimates the power of the undesired signal on each subcarrier, and the LLR is also calculated on each subcarrier, and thus, the information about the superposed band is not required. Simulation results show that the accuracy of estimating the undesired signal power becomes comparatively reliable within iteration number of five. Furthermore, the BER of the proposed scheme also becomes better as a result of iterative estimation.

Competing interests

The authors declare that they have no competing interests.

\section{Author details}

${ }^{1}$ Department of Information and Computer Science, Keio University, 3-14-1, Hiyoshi, Kohoku-ku, Yokohama 223-8522, Japan. ${ }^{2}$ NTT Network Innovation Laboratories, NTT Corporation, 1-1, Hikarinooka, Yokosuka, Kanagawa 239-0847, Japan.

Received: 31 August 2014 Accepted: 25 January 2015

Published online: 10 February 2015

\section{References}

1. J Mashino, T Sugiyama, in Proc. IEEE PIMRC'09. Total frequency utilization efficiency improvement by superposed multicarrier transmission scheme (Tokyo, Japan, 2009), pp. 1677-1681

2. T Ohtsuki, G Hayashi, J Mashino, T Sugiyama, in Proc. IEEE VTC'12-Spring. Superposed band detection based on error probability using initial likelihood masking (Yokohama, Japan, 2012), pp. 1-5

3. G Hayashi, T Ohtsuki, J Mashino, T Sugiyama, in IEEE VTS Asia Pacific Wireless Communications Symposium (APWCS2013). Interference detection 
technique based on residual power for superposed multicarrier transmission (Seoul, Korea, 2013), pp. 125-129

4. N Yoda, G Hayashi, T Ohtsuki, J Mashino, T Sugiyama, in Proc. IEEE PIMRC'13. Interference detection technique using robust LLR for superposed multicarrier transmission (London, UK, 2013), pp. 1097-1101

5. S Takaoka, H Gacanin, F Adachi, Impact of imperfect channel estimation on OFDM/TDM performance. IEEE VTC 2005-Spring. 1, 442-446 (2005)

6. X Tang, M-S Alouini, A Goldsmith, Effect of channel estimation error on M-QAM BER performance in Rayleigh fading. IEEE Trans Commun. 47(12), 1856-1864 (1999)

7. MM Wang, T Brown, Soft decision metric generation for QAM with channel estimation error. IEEE Trans. Commun. 50(7), 1058-1061 (2002)

8. H Yuan, P Kam, The LLR metric for q-ary LDPC codes with MPSK modulation over Rayleigh channels with imperfect CSI. IEEE Trans. Commun. 60(9), 1793-1799 (2012)

Submit your manuscript to a SpringerOpen ${ }^{\circ}$ journal and benefit from:

- Convenient online submission

- Rigorous peer review

- Immediate publication on acceptance

- Open access: articles freely available online

- High visibility within the field

- Retaining the copyright to your article

Submit your next manuscript at $\boldsymbol{\nabla}$ springeropen.com 